\title{
Effects of Spontaneous Lorentz Violation in Gravity
}

\author{
Robert Bluhm* \\ Colby College, Waterville, ME 04901, USA \\ E-mail: rtbluhmecolby • edu
}

\begin{abstract}
Spontaneous breaking of local Lorentz symmetry occurs when a local vector or tensor field acquires a nonzero vacuum expectation value. The effects of such breaking are examined in the context of gravity theory. These include an associated spontaneous breaking of diffeomorphism symmetry and generation of massless Nambu-Goldstone modes. The possibility of a Higgs mechanism is examined as well, and it is found that the conventional Higgs mechanism (giving rise to massive gauge fields) does not occur in a Riemann spacetime. However, in a Riemann-Cartan spacetime a Higgs mechanism involving the spin connection is possible. Despite the lack of a conventional Higgs mechanism in Riemann spacetime, additional massive modes involving the metric can appear through unconventional processes that have no analogue in nonabelian gauge theory. The effects of these types of processes are illustrated using a specific model, known as a bumblebee model, in which a vector field acquires a vacuum value.
\end{abstract}

From Quantum to Emergent Gravity: Theory and Phenomenology

June 11-15 2007

Trieste, Italy

${ }^{*}$ Speaker. 


\section{Introduction}

Interest in the possibility of Lorentz violation has increased significantly over the years, as it has been realized that effects arising at the Planck scale might give rise to small breakings of Lorentz symmetry at low energy. These include mechanisms in string theory, field theories in noncommutative geometry, quantum gravity, effects due to modified dispersion relations, etc. (For reviews, see [1, 2, 3]). At the same time, a comprehensive phenomenological investigation of Lorentz violation has been initiated that has led to a number of new high-precision tests of Lorentz invariance. This was facilitated by development of the Standard-Model Extension (SME) [4, 5], which provides the most general effective field-theoretical framework incorporating Lorentz and CPT violation. Using the SME, detailed investigations of Lorentz breaking can be conducted in the context of high-energy particle physics, gravitational physics, nuclear and atomic physics, and astrophysics. This on-going effort has pushed experimental bounds on some forms of Lorentz breaking well beyond levels associated with suppression by the Planck mass. Nonetheless, many signals remain untested. In addition to these phenomenological investigations, the idea of Lorentz breaking continues to undergo theoretical scrutiny. In particular, possible effects of Lorentz breaking in the context of gravity have begun to be explored, and in certain cases these ideas can lead to interesting prospects for alternative explanations of such things as dark matter and dark energy.

One of the more elegant ideas for Lorentz violation is that this symmetry might be spontaneously broken [6]. Indeed, it was the idea that mechanisms in string field theory might lead to spontaneous Lorentz violation that helped stimulate much of the current interest in the topic of Lorentz breaking. Moreover, one of the primary interpretations of the coefficients in the SME is that they are vacuum expectation values (combined with Yukawa couplings) of tensor fields that couple to conventional matter at low energy. Thus, one of the products of a comprehensive investigation of Lorentz violation using the SME is a survey for possible signals of spontaneous Lorentz breaking originating from the Planck scale.

However, as soon as one begins to discuss the idea of spontaneous symmetry breaking, well known results from particle physics immediately come into play. These include the possible appearance of massless Nambu-Goldstone (NG) modes, the possibility of a Higgs mechanism, and the question of whether additional massive modes (analogous to the Higgs boson) can arise.

It is these types of effects that are examined here for the case where it is local Lorentz symmetry that is spontaneously broken. Clearly, any processes generating massless or massive modes can have important implications for phenomenology. This is particularly the case for spontaneous breaking of Lorentz symmetry in the context of gravity, where the effects of the NG and massive modes might influence gravitational propagation or alter the form of the static Newtonian potential. Thus, in addition to looking at the fate of the NG modes in general and the question of whether a Higgs mechanism can occur, it is important as well to look at the role of the NG and massive modes in the context of specific models that permit an examination of their effects on gravity. The simplest example is for the case of a vector field, where such models are known as bumblebee models [6, 7]. It is this type of model that is used here to illustrate the effects of the NG and massive modes. This is then followed by a more general discussion of phenomenology.

Many of the main results presented here, including background on bumblebee models, are described in greater detail in $[6,7,8,9]$, as well as in the references cited within these works. 


\section{Spontaneous Lorentz Breaking}

In special relativity, Lorentz symmetry is a global symmetry consisting of rotations and boosts However, in curved spacetime, in a gravitational theory, Lorentz symmetry is a local symmetry. It transforms local vectors and tensors in the tangent plane at each spacetime point. In addition to being locally Lorentz invariant, a gravitational theory is also invariant under diffeomorphisms. These transformations act on tensor and vector fields defined on the spacetime manifold. Typically, it is the diffeomorphism symmetry that is more readily apparent in a gravitational theory, since local Lorentz symmetry acts only in local frames. However, ultimately both types of transformations are important, and a complete discussion of Lorentz breaking in gravity theory should include an examination of its effects on diffeomorphisms as well.

One way to reveal the transformation properties of vectors and tensors under both local Lorentz transformations and diffeomorphisms is by using a vierbein formalism. The vierbein $e_{\mu}^{a}$ relates tensor components defined with respect to a local basis, e.g., $T_{a b c}$ (where Latin indices denote components with respect to a local frame), to those defined with respect to the spacetime coordinate system, e.g., $T_{\lambda \mu \nu}$ (where Greek indices label the spacetime frame). For example, the spacetime metric and local Minkowski metric are related by

$$
g_{\mu v}=e_{\mu}^{a} e_{v}^{b} \eta_{a b}
$$

Similarly, for an arbitrary tensor,

$$
T_{\lambda \mu \nu}=e_{\lambda}^{a} e_{\mu}^{b} e_{v}^{c} T_{a b c} .
$$

A vierbein formalism also allows spinors to be incorporated into the theory, and it naturally parallels gauge theory, with Lorentz symmetry and diffeomorphisms both acting as local symmetry groups. In a vierbein formalism, the spin connection $\omega_{\mu}^{a b}$ enters in covariant derivatives that act on local tensor components and plays the role of the gauge field for the Lorentz symmetry. In contrast, the metric excitations act as the gauge fields for the diffeomorphism symmetry. When working with a vierbein formalism, there are primarily two geometries that can be distinguished. In Riemannian geometry (with no torsion), the spin connection is nondynamical. It is purely an auxiliary field that does not propagate. However, in Riemann-Cartan geometry (with nonzero torsion), the spin connection must be treated as independent degrees of freedom that in principle can propagate.

In considering theories with violation of Lorentz and diffeomorphism symmetry it is important to distinguish between observer and particle transformations [4]. Under an observer general coordinate or local Lorentz transformation, vectors and tensors remain unchanged, while the coordinate bases used to define their components transform. In contrast, particle diffeomorphisms and Lorentz transformations change vectors and tensors, while leaving unchanged the coordinate systems and basis vectors. In theories with no symmetry breaking, the transformation laws for observer and particle transformations are inversely related but otherwise are similar in form. However, if the symmetries are spontaneously broken and fields are divided into vacuum values and excitations, the transformation laws for these will differ for the observer and particle transformations.

A fundamental premise is that a physical theory should always be observer independent. This includes even when Lorentz symmetry and diffeomorphisms are either explicitly or spontaneously broken. In fact, the SME is based on this. It is formulated as a lagrangian-based field theory that 
is fully invariant under observer general coordinate transformations and local Lorentz transformations.

In a theory with spontaneous breaking of a particle spacetime symmetry, the lagrangian still remains invariant under the broken symmetry, and the full equations of motion remain covariant. However, fixed vacuum-valued fields appear that cannot be transformed under the particle transformations. Interaction terms involving these fixed background vacuum fields also appear in the equations of motion, which by themselves break the particle symmetry. It is the interaction with these vacuum fields that can lead to physical effects of the broken particle symmetry that can be tested in experiments.

In a gravitational theory, local Lorentz symmetry is spontaneously broken when a local tensor field acquires a vacuum expectation value (vev). For example, for the case of a three-component tensor,

$$
<T_{a b c}>=t_{a b c} .
$$

The vacuum of the theory then has preferred spacetime directions in the local frames, which spontaneously breaks the particle Lorentz symmetry.

Spontaneous Lorentz breaking can be introduced into a theory dynamically by adding a potential term $V$ to the Lagrangian. For example, a potential of the form

$$
V \sim\left(T_{\lambda \mu \nu} g^{\lambda \alpha} g^{\mu \beta} g^{v \gamma} T_{\alpha \beta \gamma} \pm t^{2}\right)^{2}
$$

consisting of a quadratic function of products of the tensor components $T_{\lambda \mu \nu}$, has a minimum when

$$
T_{\lambda \mu \nu} g^{\lambda \alpha} g^{\mu \beta} g^{v \gamma} T_{\alpha \beta \gamma}=\mp t^{2} .
$$

Note that the sign on the right-hand side depends on the timelike or spacelike nature of the tensor components. Solutions of Eq. (2.5) span a degenerate space of possible vacuum solutions. Spontaneous Lorentz breaking occurs when a particular vacuum value $t_{a b c}$ in the local frame is chosen, satisfying the condition

$$
\mp t^{2}=t_{a b c} \eta^{p a} \eta^{q b} \eta^{r c} t_{p q r}
$$

Alternatively, a potential with a Lagrange multipler field $\lambda$ can impose (2.5) directly as a constraint, which also leads to spontaneous selection of a vacuum value $t_{a b c}$.

\section{Nambu-Goldstone Modes}

To examine the fate of the NG modes in a theory with spontaneous Lorentz violation, a general approach can first be followed. Consider a theory with a tensor that has a nonzero vev in a local Lorentz frame, for example, $<T_{a b c}>=t_{a b c}$. Such a vev spontaneously breaks particle local Lorentz symmetry. In addition, the vierbein also has a constant or fixed background value. For example, in a background Minkowski spacetime,

$$
<e_{\mu}^{a}>=\delta_{\mu}^{a} .
$$

The spacetime tensor therefore has a vev as well,

$$
<T_{\lambda \mu v}>=t_{\lambda \mu v}
$$


which is obtained when $\left\langle e_{\mu}^{a}\right\rangle$ acts on $t_{a b c}$. This fixed vacuum value for $T_{\lambda \mu \nu}$ means that particle diffeomorphisms are spontaneously broken. Thus, a first general result is that spontaneous breaking of local Lorentz symmetry implies spontaneous breaking of diffeomorphisms.

Spontaneous breaking of these symmetries implies that NG modes should appear (in the absence of a Higgs mechanism). This raises the question of how many NG modes can appear. The usual rule is that there can be up to as many NG modes as there are broken symmetries. In this case, maximal symmetry-breaking would yield six broken Lorentz generators and four broken diffeomorphisms. Therefore, there can be up to ten NG modes in general.

A related question asks where the ten NG modes reside. In general, the answer depends on the choices of gauge. However, one natural choice is to put all ten NG modes into the vierbein, as a simple counting argument shows is possible. The vierbein $e_{\mu}^{a}$ has 16 components. With no spontaneous Lorentz breaking, the six Lorentz and four diffeomorphism degrees of freedom can be used to reduce the vierbein down to six independent degrees of freedom. (Note that a general gravitational theory can have six propagating metric modes; however, general relativity is special in that there are only two). In contrast, in a theory with spontaneous Lorentz breaking, where all ten spacetime symmetries are broken, the vierbein can have 16 propagating degrees of freedom. Therefore, a second result is that in a theory with spontaneous Lorentz breaking, up to ten NG modes can appear and all of them can naturally be incorporated as degrees of freedom in the vierbein.

These results can be obtained as well using an expansion of the vierbein in terms of infinitesimal excitations about the vacuum. For such small excitations, the distinction between local and spacetime components can be dropped (with Greek letters being used for both from here on). The vierbein (with lowered indices) is then written as

$$
e_{\mu v}=\eta_{\mu v}+\left(\frac{1}{2} h_{\mu v}+\chi_{\mu v}\right)
$$

in terms of symmetric components, $h_{\mu \nu}=h_{\nu \mu}$, and antisymmetric components, $\chi_{\mu \nu}=-\chi_{v \mu}$. Next, consider small excitations of the tensor field about its vacuum value. For the case of a threecomponent tensor, these have the form

$$
\tau_{\lambda \mu v}=\left(T_{\lambda \mu v}-t_{\lambda \mu v}\right)
$$

The NG modes are the field excitations that stay within the minimum of the potential $V$. They therefore obey the condition (2.5). A solution of this condition is given by the vierbein acting on the local vev, and is equal to

$$
T_{\lambda \mu v}=e_{\lambda \alpha} e_{\mu \beta} e_{v \gamma} t^{\alpha \beta \gamma}
$$

Inserting the expansion of the vierbein into this equation and solving for the tensor-field excitations to lowest order gives

$$
\tau_{\lambda \mu v} \approx\left(\frac{1}{2} h_{\lambda \alpha}+\chi_{\lambda \alpha}\right) t_{\mu v}^{\alpha}+\left(\frac{1}{2} h_{\mu \alpha}+\chi_{\mu \alpha}\right) t_{\lambda{ }^{\alpha}}{ }^{\alpha}+\left(\frac{1}{2} h_{v \alpha}+\chi_{v \alpha}\right) t_{\lambda \mu}{ }^{\alpha} .
$$

Evidently, it is the combination $\left(\frac{1}{2} h_{\mu \nu}+\chi_{\mu \nu}\right)$ that contains the NG fields. Indeed, with the appropriate gauge choices, an expansion of this combination as virtual local Lorentz transformations and diffeomorphisms shows explicitly that the NG modes reside in these components [8]. In this form, 
the Lorentz NG modes can be associated with the six antisymmetric components $\chi_{\mu \nu}$ while the diffeomorphism NG modes can be most closely associated with the four gauge degrees of freedom in $h_{\mu v}$.

\section{Gravitational Higgs Mechanism}

In the context of gravity, since Lorentz symmetry is a local symmetry, the possibility of a Higgs mechanism naturally arises. However, since there are two sets of broken symmetries (Lorentz and diffeomorphisms) there are potentially two associated Higgs mechanisms. Furthermore, there is also the possibility that additional massive modes can arise as field excitations obeying $V^{\prime} \neq 0$ that do not stay in the potential minimum. This section discusses these possibilities.

First, consider the case of diffeomorphisms. For this symmetry, the corresponding gauge fields are the metric (or vierbein) excitations, and therefore a conventional Higgs mechanism would presumably give rise to mass terms for the metric. However, it was previously shown that the usual Higgs mechanism involving the metric does not occur [6]. This is because in the conventional Higgs mechanism the quadratic terms (that give rise to mass terms) come from the kinetic terms for the field acquiring a vev (the tensor field in this case). These kinetic terms consist of products of covariant derivatives acting on the tensor field. However, for diffeomorphism-covariant (as opposed to gauge-covariant) derivatives it is the connection that appears in quadratic form. For example,

$$
\left(D_{\rho} T_{\lambda \mu \nu}\right)^{2} \sim\left(\Gamma_{\rho \lambda}^{\sigma} t_{\sigma \mu \nu}\right)^{2}+\cdots
$$

Since the connection consists of derivatives of the metric, and not the metric itself, there are no mass terms generated for the metric. As a result, there is no conventional Higgs mechanism for the metric.

However, it was also pointed out in Ref. [6] that the form of the potential $V$, as for example in Eq. (2.4), does permit quadratic terms involving excitations of the metric to appear. This results in an alternative form of the Higgs mechanism that has no direct analogue in nonabelian gauge theory. This is because in nonabelian gauge theory, the potential $V$ only involves the scalar Higgs fields (and not the gauge fields). However, in the case of spontaneous diffeomorphism breaking, it is combinations of both the tensor field and the metric field excitations that acquire quadratic mass terms. Since the metric appears in these terms, but not in the usual Fierz-Pauli form, it becomes possible in principle to generate mass terms that avoid the van Dam, Veltmann, and Zakharov discontinuity [10]. However, the question of whether ghost modes are generated must also be addressed. This typically becomes a model-dependent issue, since the form of the kinetic terms for the tensor fields can influence whether the massive-mode excitations propagate and whether ghost modes appear. In some models, the massive modes do not propagate, but instead remain auxiliary fields. However, even in these cases, the massive modes can have an influence on gravitational interactions, including possible modifications of the Newtonian potential [9].

Summarizing for the case of diffeomorphisms, the general results are that there is no conventional Higgs mechanism for the graviton; however, mass terms involving the metric may arise due to the form of the potential $V$, resulting in an alternative Higgs mechanism that has no direct parallel in nonabelian gauge theory. 
The next question is whether a Higgs mechanism can occur stemming from the broken local Lorentz symmetry. For the Lorentz symmetry, it is found that a conventional Higgs mechanism can occur [8]. The relevant gauge field for the Lorentz symmetry is the spin connection. It appears directly in expressions for covariant derivatives acting on local tensor components. For the local tensor that acquires a vev, quadratic mass terms for the spin connection can be generated, following the usual Higgs mechanism. For example, kinetic terms of the form

$$
\left(D_{\rho} T_{\lambda \mu \nu}\right)^{2} \sim\left(\omega_{\rho}^{\alpha}{ }_{\lambda}^{\alpha} t_{\alpha \mu \nu}\right)^{2}+\cdots
$$

can generate quadratic terms for the spin connection. However, a viable Higgs mechanism of this form involving the spin connection can only occur if the spin connection itself is a dynamical field. This requires that there is nonzero torsion and therefore that the geometry is Riemann-Cartan. Thus, a Higgs mechanism for the spin connection is possible, but only in a Riemann-Cartan geometry. Constructing a ghost-free model with a propagating spin connection is known to be a challenging problem [11]. Incorporating Lorentz violation may lead to the appearance of additional mass terms, which could create new possibilities for model building. Some preliminary investigations of this possibility have been carried out, but the search for viable models remains largely an open problem.

The discussion in this section and the previous section shows that when Lorentz symmetry is spontaneously broken there will in general be both massless NG modes and massive modes. Clearly, any theory with spontaneous Lorentz breaking must account for these modes and what their role is in the underlying dynamics described by the theory. A more definite investigation along these lines requires working in the context of a concrete model. A given model is defined by the rank of the tensor acquiring a vev and by the forms of the kinetic and potential terms. The simplest example is a theory with a vector field that has a nonzero vacuum value induced by a potential $V$. A model of this type is known as a bumblebee model $[6,7]$.

\section{Bumblebee Models}

There are numerous examples of bumblebee models that have been explored in recent years. (For some specific examples, see [6, 7, 8, 9, 12, 13, 14, 15, 16, 17, 18, 19, 20, 21, 22, 23, 24, 25, 26]). They all involve a vector field $B_{\mu}$ that acquires a fixed vacuum value $b_{\mu}$. They can be defined generally in Riemann-Cartan spacetime, or restrictions to Riemann or Minkowski spacetime can be considered. A complete definition depends on the choice of kinetic and potential terms for $B_{\mu}$ and the gravitational fields. A general Lagrangian typically has the form

$$
\mathscr{L}=\mathscr{L}_{0}-V\left(B_{\mu} B^{\mu} \pm b^{2}\right)+\mathscr{L}_{\mathrm{M}},
$$

where $\mathscr{L}_{0}$ contains the kinetic terms, $V$ is the potential that induces spontaneous Lorentz breaking, and $\mathscr{L}_{\mathrm{M}}$ contains additional interaction and matter terms. A particularly noteworthy feature that all bumblebee models share is that they do not have a local $U(1)$ gauge symmetry. This symmetry is broken explicitly by the form of the potential $V$, which in general has a functional form involving products of $B_{\mu}$,

The specific choice of kinetic terms is largely a reflection of how the bumblebee field $B_{\mu}$ is to be interpreted. One approach is to view $B_{\mu}$ as the vector in a vector-tensor theory of gravity. In this 
case, an appropriate kinetic term has a form similar to that investigated by Will and Nordvedt [27],

$$
\begin{aligned}
\mathscr{L}_{0}=\frac{1}{16 \pi G}\left[R+\sigma_{1} B^{\mu} B^{v} R_{\mu v}\right. & +\sigma_{2} B^{\mu} B_{\mu} R-\frac{1}{4} \tau_{1} B_{\mu v} B^{\mu v} \\
& \left.+\frac{1}{2} \tau_{2} D_{\mu} B_{v} D^{\mu} B^{v}+\frac{1}{2} \tau_{3} D_{\mu} B^{\mu} D_{v} B^{v}\right],
\end{aligned}
$$

where $B_{\mu \nu}=D_{\mu} B_{v}-D_{v} B_{\mu}$. A generalization of this form adds an additional fourth-order term in $B_{\mu}$ [16]. In this type of approach, it is common to assume only gravitational couplings to matter, and therefore the terms $\mathscr{L}_{\mathrm{M}}$ are not directly relevant and can be dropped.

An alternative interpretation of the vector field $B_{\mu}$ is that it is a generalized vector potential. In this case, the field strength $B_{\mu \nu}$ can be considered the more physically relevant quantity, and the natural choice of kinetic terms have an Einstein-Maxwell form, as first considered by Kostelecký and Samuel (KS) [6],

$$
\mathscr{L}_{0}^{\mathrm{KS}}=\frac{1}{16 \pi G} R-\frac{1}{4} B_{\mu v} B^{\mu \nu} .
$$

Note that there is still no local $U(1)$ gauge symmetry in this class of models when a nonzero potential $V$ is included in the full Lagrangian. However, it is common in this case to include couplings to matter along with some basic notion of charge in the matter sector. For example, terms involving current couplings with charged matter can be included by defining, $\mathscr{L}_{\mathrm{M}}=B_{\mu} J^{\mu}$ with $D_{\mu} J^{\mu}=0$. In this case, the theory has a global $U(1)$ symmetry that gives rise to charge conservation in the matter sector.

In a similar way, there are different choices that can be made for the potential $V$. One choice is a smooth quadratic potential,

$$
V=\frac{1}{2} \kappa\left(B_{\mu} B^{\mu} \pm b^{2}\right)^{2}
$$

where $\kappa$ is a constant (of mass dimension zero). This type of potential allows both NG excitations (obeying $V^{\prime}=0$ ) as well as massive excitations (obeying $V^{\prime} \neq 0$ ). A second example is a linear Lagrange-multiplier potential

$$
V=\lambda\left(B_{\mu} B^{\mu} \pm b^{2}\right),
$$

where $\lambda$ is a Lagrange-multiplier field. In this case, the Lagrange multiplier field $\lambda$ imposes a constraint, $B_{\mu} B^{\mu}=\mp b^{2}$, which only allows NG excitations in $B_{\mu}$ and excludes massive-mode excitations. However, there is still an additional degree of freedom in the form of the Lagrangemultiplier field $\lambda$, and its effects on dynamics must be understood along with those due to the NG modes.

\subsection{KS Bumblebee Model}

To illustrate the behavior of the NG and massive modes and for definiteness, consider the case of a KS bumblebee model. In the absence of a cosmological-constant term, it has a Lagrangian with the kinetic term $\mathscr{L}_{0}^{\mathrm{KS}}$ in (5.3). To allow for effects due to a massive mode, the potential $V$ is chosen as the smooth quadratic potential in (5.4). For simplicity, the vacuum value $b_{\mu}$ is taken as timelike, and an interaction of the form $B_{\mu} J^{\mu}$ is chosen. To begin the analysis, a Riemannian spacetime geometry is assumed. Generalization to a Riemann-Cartan geometry is deferred to a later section. 
The equations of motion for the KS bumblebee model are obtained by varying the Lagrangian with respect to the metric and bumblebee fields. The results are

$$
\begin{gathered}
G^{\mu v}=8 \pi G T^{\mu v}, \\
D_{v} B^{\mu v}=J^{\mu}-2 V^{\prime} B^{\mu} .
\end{gathered}
$$

Here, $G^{\mu v}$ is the Einstein tensor and $T^{\mu v}$ is the total energy-momemtum tensor, which consists of two terms,

$$
T^{\mu v}=T_{M}^{\mu v}+T_{B}^{\mu v}
$$

$T_{M}^{\mu v}$ is the energy-momentum tensor for the matter sector, while the bumblebee energy-momentum is given by

$$
T_{B}^{\mu v}=B^{\mu \alpha} B_{\alpha}^{v}-\frac{1}{4} g^{\mu v} B_{\alpha \beta} B^{\alpha \beta}-V g^{\mu v}+2 V^{\prime} B^{\mu} B^{v} .
$$

The contracted Bianchi identities for $G_{\mu \nu}$ lead to conservation of the total energy-momentum tensor,

$$
D_{\mu} T^{\mu v}=D_{\mu}\left(T_{M}^{\mu v}+T_{B}^{\mu v}\right)=0 .
$$

Similarly, the antisymmetry of the bumblebee field strength $B_{\mu \nu}$ leads to a current-conservation law following from (5.7),

$$
D_{\mu}\left(J^{\mu}-2 V^{\prime} B^{\mu}\right)=0
$$

Examination of these equations reveals that when a massive mode is present, with $V^{\prime} \neq 0$, it acts as both a source of energy and charge density. However, in the absence of a massive mode, $V^{\prime}=V=0$, and the equations reduce to the usual Einstein-Maxwell equations. Since the NG modes obey the condition $V^{\prime}=0$, these modes by themselves obey the same equations as in electrodynamics in a gravitational background. This raises the interesting possibility that massless photons arise in this type of model not as a result of gauge invariance but instead as a result of the appearance of NG modes when Lorentz symmetry is spontaneously broken.

The idea that photons might arise as NG modes due to spontaneous Lorentz breaking arose initially in the context of special relativity, where Lorentz symmetry is a global symmetry. In these early models, e.g., the model of Nambu [28], the nonzero vacuum value is imposed as a nonlinear gauge choice in the context of a theory with local U(1) gauge invariance. As a result of this, there are no physical signatures of Lorentz violation. The bumblebee models are different in that they do not have local U(1) gauge invariance. They also permit matter couplings with the vacuum value $b_{\mu}$, which can provide physical signatures of Lorentz violation.

A more complete determination of whether Einstein-Maxwell solutions can emerge from bumblebee models, requires understanding the role of the massive mode. It constitutes an additional degree of freedom beyond those of the NG modes that must be accounted for. It also alters the form of the initial-value problem. However, an exact solution of the equations of motion is not feasible, since they are highly nonlinear in form. In particular, the massive mode couples nonlinearly to both the NG and gravitational modes by acting as a source of charge and energy density. Moreover, with this extra degree of freedom present, the full nonlinear theory is known to have at least one allowed initial value (in a Minkowski-spacetime limit) for which the Hamiltonian is negative and unbounded from below $[15,16]$. It is possible, however, to restrict the theory in such a way that 
the Hamiltonian remains positive. One assumption is that the matter current $J^{\mu}$ is conserved and does not mix with the effective bumblebee charge stemming from the nonlinear field interactions. As stated above, this requires that the matter sector has a conserved charge (as expected in ordinary matter), and therefore the theory has a global $\mathrm{U}(1)$ symmetry. With this assumption, initial values can then be chosen that separate the full phase space into regions that do not mix. In particular, a region of phase space that maintains a positive Hamiltonian can be selected.

It may be possible as well to alter the stability of the theory by adding nonrenormalizable terms to the potential $V$. It has, for example, been shown (in Minkowski spacetime) that nonpolynomial potentials can lead to spontaneous Lorentz breaking and that such potentials are stable [13]. Ultimately, however, the potential instability is not likely to be relevant for physics. Viewing the bumblebee as an effective theory arising from a more fundamental and stable quantum theory of gravity, the apparent instabilities would merely reflect an incomplete knowledge of the physics entering at energy scales above that of the effective theory. However, in the absence of a fundamental quantum theory, it is not possible to pursue these questions further.

For the purposes considered here, with the aim of illustrating the effects of the NG and massive modes in a gravitational theory, it suffices to consider the bumblebee model with a KS kinetic term and conserved matter currents. It also suffices to work in the linearized limit. In such a limit, the Hamiltonian is positive (in a Minkowski-spacetime limit), while the massive mode retains its feature of behaving as a source of both charge and energy density. Hence, this limit is suitable for examining the effects of the massive mode on the gravitational interactions.

\subsection{NG and Massive Modes}

Solutions for the diffeomorphism and Lorentz NG modes can be obtained directly in the linearized approximation. With a vector vev $b_{\mu}$, symmetry under three Lorentz transformations and one diffeomorphism are spontaneously broken. Thus, there can be up to three Lorentz NG modes and one diffeomorphism NG mode. Using a vierbein formalism, the NG modes can be written as small virtual transformations away from the vacuum solution. Or, alternatively, gauge choices can be made that leave the NG modes as combinations of the bumblebee excitations $\mathscr{E} \mu=\left(B^{\mu}-b^{\mu}\right)$ and the metric excitations $h_{\mu \nu}$.

First, considering the diffeomorphism NG mode, it is found that it drops out completely from the linearized theory and does not propagate as a physical massless mode [8]. Indeed, any Lagrangian formed out of contractions of the curvature tensor and the field strength $B_{\mu \nu}$ will not contain an NG mode for the broken diffeomorphisms.

In contrast, the Lorentz NG modes are found to consist of two propagating transverse massless modes and one auxiliary mode that does not propagate [8]. They obey a condition that can be written in terms of $\mathscr{E}^{\mu}$ and $h_{\mu \nu}$ as $b^{\mu}\left(\mathscr{E}_{\mu}-\frac{1}{2} h_{\mu \nu} b^{v}\right)=0$, which resembles a type of axial-gauge condition in electromagnetism in the presence of gravity. Hence, as expected, it is found that the Lorentz NG modes behave like photons in curved spacetime. However, it must be stressed again that the bumblebee models in general have additional matter couplings that can provide physical signatures of Lorentz violation, so the NG sector coupled to matter is not strictly speaking equivalent to Einstein-Maxwell theory.

A massive mode consisting of field excitations that do not stay in the potential minimum can also occur as a solution of the equations of motion [9]. Unlike the NG modes, it cannot be written in 
terms of the vierbein and vacuum values alone, since the condition $B_{\mu} B^{\mu}=\mp b^{2}$ does not hold for the massive mode. At linear order, and in terms of $\mathscr{E}^{\mu}$ and $h_{\mu v}$, the massive mode can be identified as the combination

$$
\beta=\mp \frac{b^{\mu}\left(\mathscr{E}_{\mu}-\frac{1}{2} h_{\mu v} b^{v}\right)}{b^{2}}
$$

It is clearly independent of the Lorentz NG modes, which obey $\beta=0$.

To lowest order, the equations of motion reveal a condition the massive mode must obey,

$$
b^{\mu} \partial_{\mu} \beta \simeq 0 .
$$

For the case of a timelike vector field, with $b_{\mu}=(b, 0,0,0)$, this condition shows that the massive mode does not propagate as a free field in the linearized limit. Instead, it is purely an auxiliary field $\beta(\vec{x})$ that has no time dependence. As a result, its value is fixed by the initial conditions at $t=0$. Although it does not propagate, it can nevertheless alter the form of the static potentials.

As an example of this, consider a static point particle with mass $m$ and charge $q$. In the absence of Lorentz violation the static potentials are the usual Coulomb potential $\Phi_{q}=q / 4 \pi r$ and the Newtonian gravitational potential $\Phi_{m}=-G m / r$. Both $\Phi_{q}$ and $\Phi_{m}$ obey Poisson equations that determine the form of these potentials. Each has a source given by the point-particle mass or charge density. In the presence of spontaneous Lorentz violation, it is convenient to introduce a third potential $\Phi_{B}$ for the bumblebee massive mode. It is also defined by a Poisson equation,

$$
\vec{\nabla}^{2} \Phi_{B}(\vec{x})=-\rho_{B}
$$

where it is the massive mode $\beta(\vec{x})$ that acts as a source of density $\rho_{B}=-4 \kappa b^{2} \beta$. It is this extra degree of freedom that enters in the equations of motion and alters the form of the electromagnetic and gravitational static potentials.

Electric and magnetic fields can be defined with the usual form, but as functions of the bumblebee excitations. First define $F_{\mu \nu}=\partial_{\mu} \mathscr{E}_{\nu}-\partial_{\nu} \mathscr{E} \mu$, and solve for its components using the linearized field equations. The $\vec{E}$ and $\vec{B}$ fields can then be determined for the case of a static point particle. It is found that the fields are modified by the presence of the massive mode and are given as

$$
\vec{E}=-\vec{\nabla} \Phi_{q}-\vec{\nabla} \Phi_{B}, \quad \vec{B}=0
$$

Evidently, there is no static magnetic field generated for the case of a purely timelike vacuum value $b_{\mu}$. However, the static electric field is modified by the presence of the massive-mode potential. Even for a neutral point mass (with $q=0$ ), a nonzero massive mode can generate a nonzero electric field.

Similarly, the modified gravitational potential $\Phi_{g}$ can be determined from the field equations of motion. For the case of a point mass, it is found to have the form

$$
\Phi_{g}=\Phi_{m}-4 \pi G b \Phi_{B}
$$

Clearly, the gravitational potential is altered by the massive mode $\beta(\vec{x})$. However, the specific form that the potential takes depends on the choice of the initial value for the massive mode. This opens up the possibility of exploring modified forms of the gravitational potential in search of, for 
example, an alternative explanation of dark matter. In fact, there is considerable freedom in this approach, since the only experimental constraints are that the potential must agree with the usual Newtonian potential over probed distance scales.

Given the lack of specific experimental guidance, a natural choice of initial value would be to set $\beta=0$ at time $t=0$. In this case, $\Phi_{B}=0$, and the static potentials reduce to the usual Coulomb and Newtonian expressions. This holds true as well with a nonzero massive mode if the scale $\left|M^{2}\right|=4 \kappa b^{2}$ becomes extremely large, approaching the Planck scale, for example. Here again, the electromagnetic and gravitational potentials approach their conventional values. These results in particular reveal that the usual Einstein-Maxwell solutions (describing massless photons as well as the correct static potentials) can emerge from a theory that has no local U(1) gauge symmetry. The photons in this case are the Lorentz NG modes, and the massive mode remains extremely heavy and thus has little effect on the static potentials.

Other examples with $\Phi_{B} \neq 0$ can be considered as well. In these cases, both the static gravitational and Coulomb potentials are modified by the massive mode. One simple example is the choice $\Phi_{B}=-\Phi_{q}$. It has $\Phi_{g} \neq \Phi_{m}$, and hence has a modified Newtonian potential. The solution of the bumblebee field has the form of a total derivative $\mathscr{E}_{\mu}=\partial_{\mu} \chi$, where $\chi$ is a scalar depending on $\Phi_{m}$ and $\Phi_{q}$ [9]. The field strength $F_{\mu \nu}$ vanishes because the bumblebee density cancels the charge density $\rho_{q}$. However, the bumblebee excitations $\mathscr{E}_{\mu}$ remain nonzero through the dependence on $\Phi_{m}$. This type of solution has an unusual behavior that has been identified as potentially flawed $[15,16]$ due to the formation of shock discontinuities in $\mathscr{E}_{\mu}$. However, in fact, this behavior is to be expected. For a point charge, the singularities in $\mathscr{E}_{\mu}$ merely reflect the fact that the field has $1 / r$ dependence stemming from its dependence on $\Phi_{m}$. Indeed, the same behavior appears in the usual solutions of Einstein-Maxwell theory in an appropriately chosen gauge.

\subsection{Higgs Mechanism for the Spin Connection}

In Riemann-Cartan spacetime, the possibility of a Higgs mechanism involving the Lorentz NG modes becomes a possibility. In this mechanism it is the spin connection that gains mass terms as a result of spontaneous Lorentz breaking. As long as the spin connection is dynamical, this mechanism can in principle be viable, leading to physical massive propagating spin-connection fields. In practice, however, it is difficult to construct a model that is ghost- and tachyon-free.

An illustration is provided by the KS bumblebee model in Riemann-Cartan spacetime. In this case, when $B_{\mu}$ has a vacuum value $b_{\mu}$, the field strength $B_{\mu \nu}$ can be written in terms of the vierbein and spin connection as

$$
B_{\mu v}=\left(e_{\mu}^{\beta} \omega_{v \beta}^{\alpha}-e_{v}^{\beta} \omega_{\mu \beta}^{\alpha}\right) b_{\alpha} .
$$

When $B_{\mu \nu}$ is squared, quadratic terms in $\omega_{\mu}{ }^{\alpha}$ appear in the lagrangian, which perturbatively have the form

$$
-\frac{1}{4} e B_{\mu v} B^{\mu v} \approx-\frac{1}{4}\left(\omega_{\mu \rho v}-\omega_{v \rho \mu}\right)\left(\omega^{\mu \sigma v}-\omega^{v \sigma \mu}\right) b^{\rho} b_{\sigma} .
$$

It is these quadratic terms that allow a Higgs mechanism to occur involving absorption of the Lorentz NG modes by the spin connection.

In Ref. [8], a number of models with generalized kinetic terms for the spin connection were considered. Finding a physical model with no ghosts, however, remains an open problem. The difficulty is in finding kinetic terms describing propagating modes that are compatible with Eq. 
(5.18) as a mass term. If ghosts are permitted, then the mechanism is straightforward. For example, with a kinetic term in the gravitational sector of the form

$$
\mathscr{L}_{0, \text { grav }}=\frac{1}{4} R_{\lambda \kappa \mu v} R^{\lambda \kappa \mu v} .
$$

all the fields $\omega_{\lambda \mu v}$ with $\lambda \neq 0$ propagate as massless modes. When this is combined with the mass term (5.18), some of the propagating modes are converted to massive modes. Other examples can be studied as well, aided by decomposing the fields $\omega_{\lambda \mu \nu}$ according to their spin-parity projections. Evidently, the incorporation of spontaneous Lorentz violation in theories with torsion provides a new arena in the search for models with propagating massive modes. The challenge, however, is to find viable models that do not allow ghosts.

\section{Phenomenology of Lorentz Violation}

The effects described in this paper originating from spontaneous Lorentz breaking can provide clear signals of physical Lorentz violation. In the end, there are basically three classes of signals: those arising from NG modes, from massive modes, and from matter couplings. The phenomenological implications of each of these can be considered.

The NG modes either lead to additional gravitational modes in the vierbein that would differ from the usual forms of gravitational radiation predicted in general relativity, or in certain cases they can be interpreted as known gauge fields such as the photon or graviton [29]. However, in the latter case, there would be no observable consequences of the NG modes themselves (at least at leading order) other than the existence of the previously known massless gauge particles.

Massive modes can arise in two ways, either through a Higgs mechanism in Riemann-Cartan spacetime, or as field excitations that do not remain in the potential minumum. In either case, they could in principle be detectable as new previously unobserved propagating particles. Alternatively, however, the massive modes might remain auxiliary fields that do not propagate, as in the example of the KS bumblebee. Their influence then appears to be limited to altering the form of the relevant static potentials. In either of these scenarios, it is likely that the scale associated with the massive modes is extremely high, and therefore their observable consequences are likely to be quite small.

Any remaining signals of spontaneous Lorentz violation would involve couplings with matter fields. For example, at low energy, signals of physical Lorentz violation would occur when a tensor vev, e.g., $\left\langle T_{\lambda \mu v}\right\rangle$, couples with conventional Standard-Model or gravitational fields. As a result, any possible signal originating in this way would be identical to a signal arising in the SME. This is because the SME allows for all observer-independent violations of Lorentz symmetry involving Standard-Model and gravitational fields. It is defined as a general effective field theory at low energy, but it also provides a connection to the Planck scale through operators of nonrenormalizable dimension [12]. In many cases, it is sufficient to restrict the full SME to minimal extensions involving only, for example, power-counting renormalizable or $\mathrm{SU}(3) \times \mathrm{S}(2) \times \mathrm{U}(1)$ gauge-invariant terms. To consider experiments in atomic physics it often suffices to restrict the SME to its QED sector. Similarly, limits of the SME that include (or exclude) gravity can be defined, and in the case with gravity either a Riemann or Riemann-Cartan geometry can be assumed.

A group of theorists centered at Indiana University initiated a comprehensive phenomenological investigation of Lorentz violation more than a decade ago. These investigations span virtually 
all areas of physics. The scope of these investigations includes searching for signals of spontaneous Lorentz violation (as well as other, e.g., explicit, forms of Lorentz breaking). This work has stimulated a number of new and improved experiments. These include classic tests of Lorentz and CPT symmetry, such as $g-2$ experiments in Penning traps, Hughes-Drever experiments, modernday Michelson-Morley experiments, as well new types of tests, such as in space satellites or with astrophysical sources. Together they cover a wide range of particle sectors in the Standard Model. Some specific examples include tests with electrons [30, 31], muons [32], hadrons [33, 34], neutrinos [35], and photons [36]. Many of these efforts are on-going, with plans for attaining significantly improved sensitivities in the coming years.

\section{Summary and Conclusions}

This work has examined possible consequences of spontaneous Lorentz violation in the context of gravity. Much of the focus has been on questions concerning the fate of the NG modes, the possibility of a Higgs mechanism, and the appearance of additional massive modes. In general, it is found that in theories with spontaneous Lorentz violation, up to ten NG modes can appear. They can all be incorporated naturally in the vierbein. For the example of a KS bumblebee model, the Lorentz NG modes can propagate like photons in an axial gauge. In principle, two Higgs mechanisms can occur, one associated with broken diffeomorphisms, the other with Lorentz symmetry. However, it has been shown that a conventional Higgs mechanism (for diffeomorphisms) involving the metric does not occur. If the geometry is Riemann-Cartan, then a conventional Higgs mechanism (for the Lorentz symmetry) can occur, in which the spin connection acquires a mass. However, in a Riemann geometry, this type of Higgs mechanism is not possible. Nonetheless, an alternative type of Higgs mechanism can occur, leading to the appearance of additional massive modes involving the metric field. These can lead to altered forms of the gravitational potential. Clearly, there are numerous phenomenological questions that arise in these processes. However, all relevant signals of Lorentz breaking at low energies invloving couplings to known Standard-Model fields can be pursued comprehensively using the SME.

\section{Acknowledgments}

This work has been supported by NSF grant PHY-0554663 and by the European Science Foundation network programme "Quantum Geometry and Quantum Gravity."

\section{References}

[1] V.A. Kostelecký, ed., CPT and Lorentz Symmetry, World Scientific, Singapore, 1999; CPT and Lorentz Symmetry II, World Scientific, Singapore, 2002; CPT and Lorentz Symmetry III, World Scientific, Singapore, 2005.

[2] D. Mattingly, Living Rev. Rel. 8, 5 (2005).

[3] J. Ehlers and C. Lämmerzahl, eds., Special Relativity: Will It Survive the Next 101 Years? eds., (Springer, Berlin, 2006). 
[4] V.A. Kostelecký and R. Potting, Phys. Rev. D 51, 3923 (1995); D. Colladay and V.A. Kostelecký, Phys. Rev. D 55, 6760 (1997); Phys. Rev. D 58, 116002 (1998).

[5] For a review of the SME, see the contribution by R. Bluhm in [3], hep-ph/0506054.

[6] V.A. Kostelecký and S. Samuel, Phys. Rev. D 40, 1886 (1989); Phys. Rev. D 39, 683 (1989); Phys. Rev. Lett. 63, 224 (1989).

[7] V.A. Kostelecký, Phys. Rev. D 69, 105009 (2004).

[8] R. Bluhm and V.A. Kostelecký, Phys. Rev. D 71, 0065008 (2005).

[9] R. Bluhm, S.-H. Fung. and V.A. Kostelecký, arXiv:0712.4119.

[10] H. van Dam and M. Veltman, Nucl. Phys. B 22, 397 (1970); V.I. Zakharov, JEPT Lett. 12, 312 (1970).

[11] E. Sezgin and P. van Nieuwenhuizen, Phys. Rev. D 21, 3269 (1980); K. Fukuma, Prog. Theor. Phys. 107, 191 (2002).

[12] V.A. Kostelecký and R. Lehnert, Phys. Rev. D 63, 065008 (2001).

[13] B. Altschul and V.A. Kostelecký, Phys. Lett. B 628, 106 (2005).

[14] V.A. Kostelecký and Q.G. Bailey, Phys. Rev. D 74, 045001 (2006).

[15] M.A. Clayton, gr-qc/0104103.

[16] C. Eling, T. Jacobson, and D. Mattingly, gr-qc/0410001.

[17] P. Kraus and E.T. Tomboulis, Phys. Rev. D 66, 045015 (2002).

[18] J.W. Moffat, Intl. J. Mod. Phys. D 12, 1279 (2003).

[19] B.M. Gripaios, JHEP 0410, 069 (2004).

[20] S.M. Carroll and E.A. Lim, Phys. Rev. D 70, 123525 (2004).

[21] O. Bertolami and J. Paramos, Phys. Rev. D 72, 044001 (2005).

[22] J.W. Elliott, G.D. Moore, and H. Stoica, JHEP 0508, 066 (2005).

[23] M.V. Libanov and V.A. Rubakov, JHEP 0508, 001 (2005).

[24] D.S. Gorbunov and S.M. Sibiryakov, JHEP 0509, 082 (2005).

[25] A.T. Azatov and J.L. Chkareuli, Phys. Rev. D 73, 065026 (2006); J.L. Chkareuli, C.D. Froggatt, and H.B. Nielsen, hep-th/0610186.

[26] H.-C. Cheng, M. Luty, S. Mukohyama, and J. Thaler, JHEP 0605, 076 (2006).

[27] C.M. Will, Theory and Experiment in Gravitational Physics, Cambridge University Press, Cambridge, England, 1993.

[28] Y. Nambu, Prog. Theor. Phys. Suppl. Extra 190 (1968).

[29] V.A. Kostelecký and R. Potting, Gen. Rel. Grav. 37, 1675 (2005).

[30] R. Bluhm et al., Phys. Rev. Lett. 79, 1432 (1997); Phys. Rev. D 57, 3932 (1998); H. Dehmelt et al., Phys. Rev. Lett. 83, 4694 (1999); R. Mittleman et al., Phys. Rev. Lett. 83, 2116 (1999); G. Gabrielse et al., Phys. Rev. Lett. 82, 3198 (1999);

[31] R. Bluhm and V.A. Kostelecký, Phys. Rev. Lett. 84, 1381 (2000); L.-S. Hou, W.-T. Ni, and Y.-C.M. Li, Phys. Rev. Lett. 90, 201101 (2003); B. Altschul, Phys. Rev. D 72, 085003 (2005); Phys. Rev. Lett. 96, 201101 (2006); Phys. Rev. D 74, 083003 (2006); B. Heckel et al., Phys. Rev. Lett. 97, 021603 (2006); 
[32] R. Bluhm et al., Phys. Rev. Lett. 84, 1098 (2000). V.W. Hughes et al., Phys. Rev. Lett. 87, 111804 (2001); M. Diele et al., in V.A. Kostelecký, ed., CPT and Lorentz Symmetry II, World Scientific, Singapore, 2002;

[33] V.A. Kostelecký and C.D. Lane, Phys. Rev. D 60, 116010 (1999); J. Math. Phys. 40, 6245 (1999); R. Bluhm et al., Phys. Rev. Lett. 82, 2254 (1999); Phys. Rev. Lett. 88, 090801 (2002); Phys. Rev. D 68, 125008 (2003); V.W. Hughes, H.G. Robinson, and V. Beltran-Lopez, Phys. Rev. Lett. 4 (1960) 342; R.W.P. Drever, Philos. Mag. 6 (1961) 683; J.D. Prestage et al., Phys. Rev. Lett. 54 (1985) 2387; S.K. Lamoreaux et al., Phys. Rev. A 39 (1989) 1082; T.E. Chupp et al., Phys. Rev. Lett. 63 (1989) 1541; C.J. Berglund et al., Phys. Rev. Lett. 75 (1995) 1879; D. Bear et al., Phys. Rev. Lett. 85, 5038 (2000); D.F. Phillips et al., Phys. Rev. D 63, 111101 (2001); M.A. Humphrey et al., Phys. Rev. A 62, 063405 (2000); F. Cane et al., Phys. Rev. Lett. 93, 230801 (2004); P. Wolf et al., Phys. Rev. Lett. 96, 060801 (2006).

[34] D. Colladay and V.A. Kostelecký, Phys. Lett. B 344, 259 (1995); Phys. Rev. D 52, 6224 (1995); Phys. Lett. B 511, 209 (2001); V.A. Kostelecký and R. Van Kooten, Phys. Rev. D 54, 5585 (1996); O. Bertolami et al., Phys. Lett. B 395, 178 (1997); V.A. Kostelecký, Phys. Rev. Lett. 80, 1818 (1998); Phys. Rev. D 61, 016002 (2000); 64, 076001 (2001); N. Isgur et al., Phys. Lett. B 515, 333 (2001); B. Altschul, Phys. Rev. D 75023001 (2007); OPAL Collaboration, R. Ackerstaff et al., Z. Phys. C 76, 401 (1997); BELLE Collaboration, K. Abe et al., Phys. Rev. Lett. 86, 3228 (2001); KTeV Collaboration, H. Nguyen, in V.A. Kostelecký, ed., CPT and Lorentz Symmetry II, World Scientific, Singapore, 2002; FOCUS Collaboration, J.M. Link et al., Phys. Lett. B 556, 7 (2003); BaBar collaboration, B. Aubert et al., Phys. Rev. D 70, 012007 (2004); Phys. Rev. Lett. 92, 142002 (2004); hep-ex/0607103.

[35] V.A. Kostelecky and M. Mewes, Phys. Rev. D 69016005 (2004); 70031902 (2004); 70076002 (2004); LSND Collaboration, L.B. Auerbach et al., Phys. Rev. D 72076004 (2005); M.D. Messier (Super Kamiokande), in V.A. Kostelecký, ed., CPT and Lorentz Symmetry III, World Scientific, Singapore, 2005; B.J. Rebel and S.F. Mufson (MINOS), in V.A. Kostelecký, ed., CPT and Lorentz Symmetry III, World Scientific, Singapore, 2005; T. Katori, A. Kostelecky, and R. Tayloe, Phys. Rev. D 74105009 (2006).

[36] V.A. Kostelecký and M. Mewes, Phys. Rev. Lett. 87, 251304 (2001); Phys. Rev. D 66, 056005 (2002); Phys. Rev. Lett. 97, 140401 (2006); J. Lipa et al., Phys. Rev. Lett. 90, 060403 (2003); H. Müller et al., Phys. Rev. Lett. 91, 020401 (2003); H. Müller et al., Phys. Rev. D 67, 056006 (2003); Phys. Rev. D 68, 116006 (2003); P. Wolf et al., Gen. Rel. Grav. 36, 2352 (2004); P. Wolf et al., Phys. Rev. D 70, 051902 (2004); H. Müller, Phys. Rev. D 71, 045004 (2005); P.L. Stanwix et al., Phys. Rev. Lett. 95, 040404 (2005); Phys. Rev. D 74, 081101 (2006); S. Herrmann et al., Phys. Rev. Lett. 95, 150401 (2005); P. Antonini et al., Phys. Rev. A 71, 050101 (2005); M.E. Tobar et al., Phys. Rev. D 71, 025004 (2005). 